\title{
Engineering Topological Many-Body Materials in Microwave Cavity Arrays
}

\author{
Brandon M. Anderson, Ruichao Ma, Clai Owens, David I. Schuster, and Jonathan Simon \\ The James Franck Institute and Department of Physics, University of Chicago, \\ Chicago, Illinois 60637, USA
}

(Received 10 May 2016; revised manuscript received 22 September 2016; published 1 December 2016)

\begin{abstract}
We present a scalable architecture for the exploration of interacting topological phases of photons in arrays of microwave cavities, using established techniques from cavity and circuit quantum electrodynamics. A time-reversal symmetry-breaking (nonreciprocal) flux is induced by coupling the microwave cavities to ferrites, allowing for the production of a variety of topological band structures including the $\alpha=1 / 4$ Hofstadter model. To induce photon-photon interactions, the cavities are coupled to superconducting qubits; we find these interactions are sufficient to stabilize a $\nu=1 / 2$ bosonic Laughlin puddle. Exact diagonalization studies demonstrate that this architecture is robust to experimentally achievable levels of disorder. These advances provide an exciting opportunity to employ the quantum circuit toolkit for the exploration of strongly interacting topological materials.
\end{abstract}

DOI: 10.1103/PhysRevX.6.041043

\section{INTRODUCTION}

Despite significant interest in topological physics, the experimental success in realizing strongly correlated topological systems has thus far been limited to the fractional quantum Hall $(\mathrm{FQH})$ effect $[1,2]$ in two-dimensional electron gases. There has recently been significant theoretical explorations of alternative realizations of $\mathrm{FQH}$ systems (and related fractional Chern insulators [3,4]) in the field of quantum engineering. Here, a topologically nontrivial Hamiltonian is built from the ground up, typically following a two-ingredient recipe [3,4]: (1) strong interactions are added to a (2) topologically nontrivial single-particle band structure. This recipe is well motivated theoretically, and experimentally there has been success in individual implementation of each ingredient. For example, ultracold atomic systems have experimentally succeeded in the study of both topologically novel band structures [5-10] and strongly interacting but topologically trivial systems $[11,12]$. However, the simultaneous implementation of both elements, as is necessary for creating strongly correlated topological states, has thus far remained elusive.

Photons are a newly emerging platform for quantum engineering. Photonic crystals have successfully simulated noninteracting band structures in the regime of rf [13], microwave [14], or optical $[15,16]$ domains. In all cases, the necessary addition of strong interactions to produce strongly correlated states has remained challenging. On the other hand, strong photon-photon interactions are

Published by the American Physical Society under the terms of the Creative Commons Attribution 3.0 License. Further distribution of this work must maintain attribution to the author(s) and the published article's title, journal citation, and DOI.
Subject Areas: Condensed Matter Physics, Metamaterials, Photonics readily achieved in circuit QED experiments where the exquisite control over few qubit states has allowed the quantum simulation of, e.g., molecular energies [17]. Superconducting circuits have also been advanced as a route to strongly correlated photonic lattices [18,19]. Implementing a time-reversal symmetry-breaking (TRSB) single-particle band structure is still necessary to advance towards FQH physics. Current proposals are experimentally difficult to scale to larger system sizes within necessary error bounds, since they require either a sitedependent parametric modulation [20] or extreme suppression of gate-charge noise [19]. Breaking time-reversal symmetry with passive and low-noise circuit elements would therefore be a significant advance in engineering of synthetic quantum materials.

Here, we propose a new photonic platform to engineer two-dimensional tight-binding models with nontrival band topology, using arrays of three-dimensional microwave cavities. Such cavities can be easily machined from metal to work in the few-to-tens of $\mathrm{GHz}$ regime, and have been shown to provide exceptional coherence at cryogenic temperatures with quality factors exceeding $Q>10^{8}$ [21]. The cavities can be tunnel coupled evanescently by, e.g., directly milling a channel between two cavities, or using capacitively coupled transmission lines. Modern machining techniques allow the creation of scalable lattices with low disorder in on-site energies and tunneling energies.

A key component of our scheme is a new technique used to induce the requisite TRSB flux: this flux is induced by the on-site mode structure [22] of the cavity, in contrast to previous schemes $[13,16,18,23]$ that induce a Peierl's phase in the tunnel coupler. The complex amplitude of the tunnel coupler depends on the local electric field at the periphery of the cavity. A nonreciprocal (TRSB) phase will require 
an electric field with on-site angular momentum, which is coupled to the polarization of the magnetic field through Faraday's law [24]. Therefore, by coupling the magnetic field to a magnetic dipole, say, through use of a ferrimagnetic crystal with a biased magnetic field [25,26], a cavity mode with definite angular momentum can be energetically isolated. In this way, the ferrite transfers the TRSB bias field to a TRSB flux of the photonic lattice. This technique is experimentally advantageous compared to previous schemes because (1) it allows for the passive creation of topological band structures, and therefore avoids issues with nonlinear mixing with pumping frequency in modulated tunnel couplings schemes [20], (2) it does not require gate-charge biasing and therefore avoids large charge noise [19], and (3) the ferrite couples to a chiral "bright" mode, shifting it in energy, leaving an unshifted chiral "dark" mode. The frequency of the dark mode is first-order insensitive to the ferrite coupling strength, loss, and detuning from cavity resonance [27]; it is thus ideal for engineering low-disorder time-reversal breaking lattice models.

The final ingredient necessary for the study of $\mathrm{FQH}$-like physics is strong photon-photon interactions. These can be incorporated by coupling the microwave cavities to superconducting qubits [18,28]. We consider adding such qubits to each site of the square Hofstdater model constructed from the linear circuit elements. The Hamiltonian describing the effective model can be simulated using exact diagonalization techniques. We therefore numerically explore our system at finite size and for few photons. The numerical results demonstrate our architecture will have a FQH eigenstate corresponding to a bosonic $\nu=1 / 2$ Laughlin state on a lattice $[9,29]$.

In the remainder of this paper, we present a scalable architecture for the study of strongly correlated topological materials. The organization of the paper is as follows. In Sec. II, we discuss the general structure of the microwave cavities needed for our architecture. We then introduce onsite coupling elements (Sec. II A), which isolate a desired eigenmode on each lattice site. Tunnel coupling the microwave cavities (Sec. II B) results in an effective flux when the system is probed in a certain frequency range. This effective flux can be understood as an emergent gauge field (Sec. II C) arising from band projection. Concluding the discussion of the general noninteracting circuit elements, we show this architecture is sufficient to simulate the Hofstadter model (Sec. II D), and present proposals for some other interesting lattice models (Sec. II E). In Sec. III, we then consider the effects of adding Josephson junction qubits to each site for the purpose of producing fractional Chern insulating states in finite-size systems. We use a numerical exact diagonalization technique to simulate our system, and find that the properties of a $\nu=1 / 2$ bosonic "Laughlin puddle" emerge. Finally, in Sec. III B, we consider the likely forms of disorder in the effective tight-binding model description of our system. We find that the system is insensitive to the largest sources of disorder, whereas the most sensitive forms of disorder can be controlled. We conclude that current experimental techniques should be sufficient to simulate strongly correlated topological systems.

\section{SINGLE-PARTICLE BUILDING BLOCKS}

We now describe the single-particle building blocks for our microwave architecture. Our goal is to engineer an effective tight-binding Hamiltonian $\mathcal{H}_{\text {eff }}$ for photons with energy near $\hbar \omega_{0}$. We use a unique cavity eigenmode at this characteristic energy to represent the tight-binding degree of freedom (and thus restrict our work to "spinless" models). To model $\mathcal{H}_{\text {eff }}$, we therefore need an isolated eigenmode on every site, whose frequency is $\omega_{0}$, with all other on-site eigenmodes far detuned energetically.

There is significant freedom in the cavity mode structure. We describe a particular implementation employing TM modes of cylindrical cavities with nonzero transverse magnetic field $\left(B_{x}, B_{y} \neq 0, B_{z}=0\right)$ and longitudinal electric field $\left(E_{x}, E_{y}=0, E_{z} \neq 0\right)$. We first consider a fundamental cavity mode tuned to frequency $\omega_{0}$ (annihilated by $a_{0}$ ). The electric field of the fundamental mode, shown in Fig. 1(a), is nodeless and has a spatially uniform phase across the cavity. This is true regardless of the cavity geometry. We also consider a different cavity where the twofold degenerate set of first excited modes (for example, $\mathrm{TM}_{210}, \mathrm{TM}_{120}$ in a Cartesian basis [30]) is tuned to a frequency $\omega_{0}$. We define the annihilation operators of this manifold as $a_{x}\left(a_{y}\right)$, according to a node of the electric field along the $\hat{y}(\hat{x})$ axis [see Fig. 1(a)]. Using this notation, the on-site Hamiltonian is $\mathcal{H}^{(0)}=\omega_{0} a_{0}^{\dagger} a_{0}$, for a fundamental mode cavity, and $\mathcal{H}^{(1)}=\omega_{0}\left(a_{x}^{\dagger} a_{x}+a_{y}^{\dagger} a_{y}\right)$ for the excited cavity manifold. Here, and in what follows, we drop any constants resulting from zero point motion of the electromagnetic field and set $\hbar=1$.

While we write the Hamiltonian in terms of $\hat{x} / \hat{y}$ modes, this choice is arbitrary. Analogous to linearly polarized light, we may rotate the basis by an angle $\theta: a_{\theta}=a_{x} \cos \theta+a_{y} \sin \theta$ and $a_{\theta+\pi / 2}=-a_{x} \sin \theta+a_{y} \cos \theta$. Alternatively, we can construct a basis analogous to circularly polarized light: $a_{ \pm}=\left(a_{x} \pm i a_{y}\right) / \sqrt{2}$, where the phase changes continuously by $2 \pi$ going counterclockwise (clockwise) around the cavity center in the $a_{+}\left(a_{-}\right)$mode [see Fig. 1(a)]. In this basis, the magnetic field has an amplitude maximum at the center of the cavity and a polarization that lies in plane and rotates uniformly in time at frequency $\omega_{0}$.

\section{A. On-site symmetry breaking}

The on-site symmetry can be broken in a time-reversal preserving or time-reversal breaking manner. The first type takes the form of violations of cylindrical symmetry. 
(a)
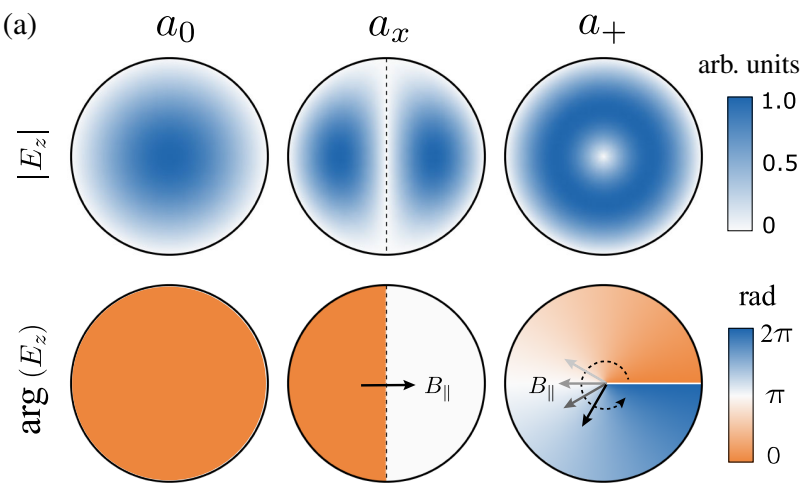

(b)
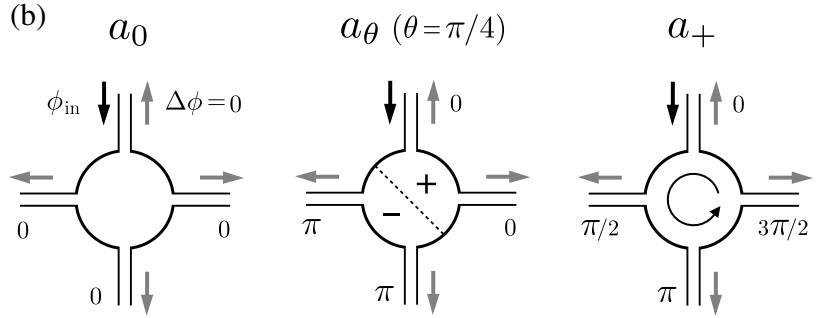

FIG. 1. (a) Structure of the electric field amplitude and phase (with corresponding magnetic field polarization) of the modes being considered. The fundamental mode has uniform phase everywhere, with no nodes in the sole electric field component $E_{z}$. The twofold degenerate first excited manifold may be spanned by two modes with geometrically orthogonal linear nodes in the $E_{z}$ field $\left(a_{x}\right.$ and $a_{y}$ ), or, equivalently, by two modes exhibiting a single point node and $\pm 2 \pi$ phase winding around the periphery $\left(a_{ \pm}\right)$. For the modes $a_{x}$ and $a_{+}$, the polarization of the magnetic field at the cavity center is presented. The polarization of the magnetic field points along the gradient of the phase of $E_{z}$. For linear modes, the magnetic field is oriented perpendicular to the nodal line, with direction oscillating in time. For the chiral modes, this magnetic field polarization precesses in time with frequency $\omega_{0}$. The size of the circles does not reflect the physical dimension of the cavities, which is chosen such that the desired mode is always at frequency $\omega_{0}$. (b) The geometric phase acquired, for a photon of frequency $\omega_{0}$, when tunneling through a cavity in which each type of mode has been isolated. Left: A fundamental mode cavity will induce no tunneling phase shift between any two contacts. Center: An isolated linear mode (via a diagonal conductor or dielectric) will induce a tunneling phase shift of either 0 or $\pi$. Right: An isolated circular mode (via a ferrite in a magnetic field) will produce a phase shift equal to the relative angle between the incoming and outgoing tunnel contacts.

For example, by placing a thin barrier (e.g., a rectangular conductor or dielectric) along the nodal line of the $a_{x}$ mode, one adds a perturbation $\mathcal{V}^{(1)}=\Delta_{\text {lin }} a_{y}^{\dagger} a_{y}$ to the Hamiltonian $\mathcal{H}^{(1)}$. The $a_{x}$ mode remains (nearly) unperturbed at frequency $\omega_{0}$, while the orthogonal $a_{y}$ mode will be shifted to higher energy (by an amount $\Delta_{\text {lin }}$ ). This energetically isolates the $a_{x}$ mode at an energy $\omega_{0}$, and uniquely defines the relative phase between any two points on the edge of the cavity. (An analogous procedure isolates $a_{\theta}$ from $a_{\theta+\pi / 2}$.)
This perturbation produces only a relative phase of 0 or $\pi$ between points on a cavity edge, and therefore cannot break time-reversal symmetry. Manufacturing imprecision gives rise to perturbations of this type.

For the study of quantum Hall physics, it is necessary to break time-reversal symmetry; this does not occur naturally for light. We consider achieving this by employing the coherent magnon interaction between cavity photons and the spins of a ferrimagnetic material $[25,26]$ : a small ferrite sphere placed at the center of the cavity with a dc bias field $\mathbf{B}=+B_{0} \hat{z}$. The ferrite acts as a collective spin that couples to the magnetic field of the cavity mode and precesses at a frequency $\omega_{F}=\mu_{0} B_{0}$. (Reversing the bias field results in an identical analysis with $a_{+} \leftrightarrow a_{-}$.) The magnetic field of the $a_{ \pm}$modes has a maximum at the cavity center (at the node of the electric field) and an in-plane polarization that precesses about $\pm \hat{z}$ at a frequency $\omega_{0}$. When the spin precession frequency $\omega_{F}$ is tuned near $\omega_{0}$, the polarization of the magnetic field of the $a_{ \pm}$mode rotates synchronously with the collective spin. The magnetic dipole interaction results in a strong coupling between the $a_{+}$mode and the ferrite mode (denoted $a_{F}$ ). The magnetic field of the $a_{-}$mode, on the other hand, rotates against the collective spin, and thus does not couple. This results in strong hybridization of the $a_{+}$mode with the ferrite, producing two bright magnon modes that are pushed away from the dark $a_{-}$mode at frequency $\omega_{0}$. Thus, when the system is probed near $\omega_{0}$, the isolated dark mode $a_{-}$sets a unique magnetic field polarization vector, or, equivalently, a unique quantum mechanical phase at the cavity periphery.

The coupling of the cavity-ferrite system is described by the Hamiltonian $\mathcal{H}^{(F)}=\mathcal{H}^{(1)}+\omega_{F} a_{F}^{\dagger} a_{F}+g_{F}\left(a_{+}^{\dagger} a_{F}+a_{F}^{\dagger} a_{+}\right)$, where $g_{F}$ is the coupling of the ferrite to the $a_{+}$mode (and must overwhelm $\left.\Delta_{\text {lin }}\right)$. A more suggestive notation is $\mathcal{H}^{(F)}=\left(\boldsymbol{a}^{(F)}\right)^{\dagger} \boldsymbol{H}^{(F)} \boldsymbol{a}^{(F)}$, where we define a ferrite-cavity mode vector $\boldsymbol{a}^{(F)}=\left(a_{x}, a_{y}, a_{F}\right)^{T}$ and describe the cavityferrite system with a coupling matrix:

$$
H^{(F)}=\omega_{0} \hat{I}+\left(\begin{array}{ccc}
0 & 0 & g_{F} / \sqrt{2} \\
0 & 0 & i g_{F} / \sqrt{2} \\
g_{F} / \sqrt{2} & -i g_{F} / \sqrt{2} & 2 \delta_{F}
\end{array}\right) \text {, }
$$

where $2 \delta_{F}$ is the detuning of the ferrite from cavity resonance $\left(\omega_{F}=\omega_{0}+2 \delta_{F}\right)$ and $\hat{I}$ is the identity matrix in the cavityferrite space. This Hamiltonian has an uncoupled dark eigenmode $a_{-}$that remains at frequency $\omega_{0}$. There are also two hybridized magnon modes $B_{ \pm}=\left(a_{F} \pm a_{+}\right) / \sqrt{2}$ that are frequency shifted by $\omega_{B_{ \pm}}-\omega_{0}=\delta_{F} \pm \sqrt{\delta_{F}^{2}+g_{F}^{2}}$. We henceforth assume the experimentally relevant limit where $g_{F}$ is large compared to $\delta_{F}$, along with any energy scales appearing in $\mathcal{H}_{\text {eff }}$.

Key features of this system are understood by examining the cavity response using input-output formalism [25,31]: 
We consider response at a small detuning $\delta_{p}=\omega-\omega_{0}$, and ferrite detuning $2 \delta_{F}$, from $\omega_{0}$, and introduce a finite lifetime of the cavity modes $(\kappa)$ and ferrite mode $\left(\kappa_{F}\right)$, with a resulting cooperativity $\eta=4 g_{F}^{2} / \kappa \kappa_{F}$. The amplitude of the response is shown in Fig. 2(a). Here, there are three maxima in the response as $\delta_{p}$ is varied, corresponding to three eigenmodes: the $a_{-}$mode remains at $\omega_{0}$ for all ferrite detunings, while the $a_{+}$mode mixes with the ferrite mode $a_{F}$ and undergoes an avoided crossing as the ferrite detuning is swept. When probed at frequencies near $\omega_{B_{ \pm}}$, the bright magnon modes have a TRSB phase winding. Importantly, while only the bright modes couple to the ferrite, both the bright and dark modes produce nonreciprocal phase shifts. The dark mode is then advantageous because it is insensitive to loss in the ferrite and is less sensitive to detuning and linear disorder. When the ferrite is exactly on resonance $\left(\delta_{F}=0\right)$, the dark mode has an exact TRSB phase winding of $2 \pi$ at the periphery of the cavity. At probe frequencies different than $\omega_{0}$, the phase winding ceases to be uniform. However, this nonuniformity is quadratically insensitive in both the ferrite detuning and the energy of the probe photon. This can be seen from the saddle-point structure in Fig. 2(b). Here, the nonreciprocal phase response of this system is calculated between two cavity contacts separated by an angle of $\pi / 4$ on the cavity periphery [inset of Fig. 2(b)]; the difference between forward and backwards phases is $\phi_{\text {nonrecip }} \approx(3 \pi / 2)-$ $2\left[\delta_{p}\left(\delta_{p}-2 \delta_{F}\right) / g_{F}^{2}\right]-(2 / \eta)$. This quadratic insensitivity of the eigenmode structure significantly decreases disorder in $\mathcal{H}_{\text {eff }}$ arising from disorder in the ferrite, cavity, or bias field.

We now define our on-site Hamiltonian at a site $j$ with the notation $\mathcal{H}_{\mathrm{os}, j}^{(\alpha)}=\left(\boldsymbol{a}_{j}^{(\alpha)}\right)^{\dagger} \boldsymbol{H}_{j}^{(\alpha)} \boldsymbol{a}_{j}^{(\alpha)}$, where $\alpha=0$ corresponds to a fundamental cavity, $\alpha=\theta$ defines a site isolating a linear mode $a_{\theta}$, and $\alpha=F$ corresponds to a ferrite site. The form of the vector of annihilation operators on site $j$ depends on the type of site with $\boldsymbol{a}_{j}^{(0)}=a_{j 0}$, $\boldsymbol{a}_{j}^{(\theta)}=\left(a_{j x}, a_{j y}\right)^{T}$, and $\boldsymbol{a}_{j}^{(F)}=\left(a_{j x}, a_{j y}, a_{j F}\right)^{T}$ for a fundamental, linear, and ferrite site, respectively. The on-site coupling Hamiltonians are $H_{j}^{(0)}=\omega_{0}$,

$$
H_{j}^{(\theta)}=\left(\begin{array}{cc}
\omega_{0} & -\Delta_{\operatorname{lin}} e^{i \theta} \\
-\Delta_{\operatorname{lin}} e^{-i \theta} & \omega_{0}
\end{array}\right),
$$

and $H_{j}^{(F)}$ is given by Eq. (1). A system of uncoupled cavities is then generically described by the on-site Hamiltonian $\mathcal{H}_{\mathrm{os}}=\sum_{j}=\sum_{j}\left(\boldsymbol{a}_{j}^{(\alpha)}\right)^{\dagger} H_{j}^{(\alpha)} \boldsymbol{a}_{j}^{(\alpha)}$, where each $\alpha_{j}$ represents one of the distinct cavity types.

\section{B. Tunnel coupling and nontrivial flux}

Coupling of the cavities is realized by connecting their edges with evanescent waveguides. The amplitude of such
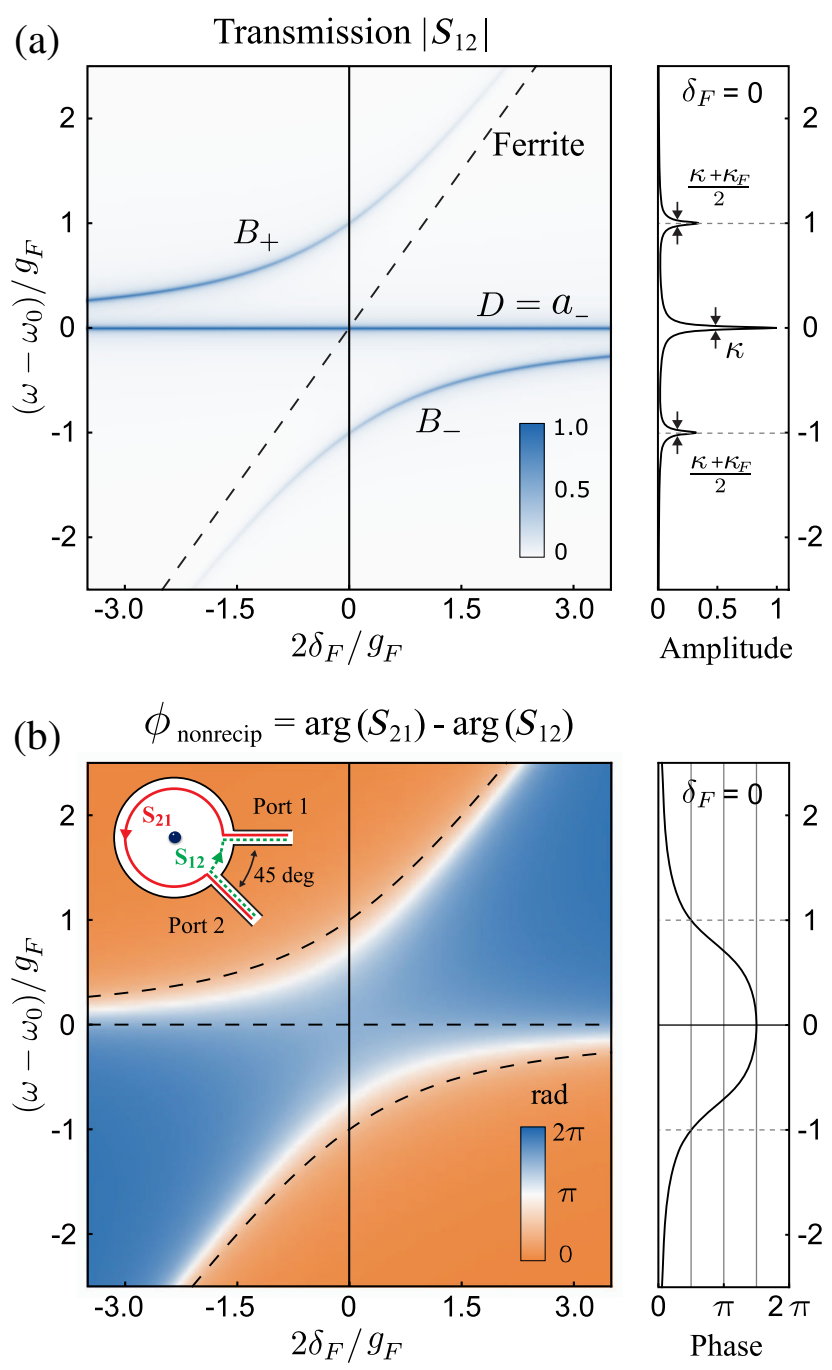

FIG. 2. Response of a ferrite-cavity site described by $\mathcal{H}^{(F)}$ in a pump-probe experiment. Here, $S_{i j}$ is the response between a port $i=1$ located at the $\hat{x}$ axis and a second port $j=2$ measured $45^{\circ}$ away [inset of (b)]. In order to account for loss, we introduce a linewidth $\kappa$ and $\kappa_{F}$ for the microwave cavity and ferrite, respectively. For physically relevant values of $\kappa / g_{F}, \kappa_{F} / g_{F}<10^{-2}$, this gives a corresponding cooperativity $\eta=4 g_{F}^{2} / \kappa \kappa_{F}>10^{4}$. (a) Amplitude of the transmission response $\left|S_{12}\right|$. The chirality of a ferrite in a magnetic field allows it to couple only to the resonator mode of the same handedness, producing an avoided crossing in the transmission spectrum as the ferrite is tuned through resonance with the resonator modes. This avoided crossing appears as two modes that are bright to the ferrite, $B_{+}$and $B_{-}$. The dark mode $D=a_{-}$does not couple to the ferrite, and is therefore unshifted and insensitive to ferrite loss $\kappa_{F}$. (b) The nonreciprocal phase, $\phi_{\text {nonrecip }}=\arg \left(S_{21}\right)-\arg \left(S_{12}\right)$, reflects the phase difference between going in the $0^{\circ}$ port and out the $45^{\circ}$ port, and the reverse process. This is ideally $\phi_{\text {nonrecip }}=3 \pi / 2$ for the dark mode. This time-reversal break is robust to detuning in either the ferrite or the probe, and also loss, with the lowest-order correction entering as $\phi_{\text {nonrecip }} \approx(3 \pi / 2)-2\left[\delta_{p}\left(\delta_{p}-2 \delta_{F}\right) / g_{F}^{2}\right]-(2 / \eta)$. (c) A slice through the nonreciprocal phase as a function of the probe detuning, for the ferrite tuned to resonance. The nonreciprocal phase of $\phi_{\text {nonrecip }} \approx 3 \pi / 2$ is apparent near the dark mode. 
a tunnel coupling is determined by the geometry (length and cross section) of the channel. We emphasize that the coupler is only virtually populated - the evanescent wave "propagates" with a purely imaginary wave vector-so the phase of the photon is spatially uniform throughout the channel, and the channel has no dynamical degree of freedom.

Now consider a site with an isolated eigenmode (such as $a_{\theta}$ or $\left.a_{ \pm}\right)$at frequency $\omega_{0}$, that has a nonuniform phase profile. Attached to this cavity are two or more tunnel contacts to nearby cavities. The nonuniform phase of the mode implies that a photon that tunnels in along one channel will acquire a geometrical phase shift as it tunnels out along a different channel; this is the origin of the induced TRSB flux.

In a fundamental mode cavity, both the amplitude and phase around the edge are uniform, and therefore no geometric phase will be acquired for two tunneling contacts, regardless of where they are attached. In contrast, a ferrite-cavity site with two tunneling channels separated by an angle $\phi$ on the cavity perimeter will experience a geometrical phase shift of $\pm \phi$ for an isolated $a_{ \pm}$mode [see Fig. 1(b)].

On the other hand, two tunnel contacts on a site with a mode $a_{\theta}$ will experience a relative phase shift only if the photon crosses a node between the processes of tunneling in and tunneling out [see Fig. 1(b)]. For an $a_{\theta}$ mode, the local amplitude at the cavity periphery will be nonuniform and the tunneling magnitude will gain position dependence [see Fig. 1(a)]. [An ideal point contact at an angle $\phi$ relative to the $\hat{x}$ axis will tunnel with amplitude $t \sim|\cos (\theta-\phi)|$ at frequencies near $\omega_{0}$.] This is in contrast to a mode $a_{0}$ or $a_{ \pm}$, for which the mode magnitude, and thus the tunneling magnitude, is uniform.

For an open 1D chain of tunnel-coupled cavities, the geometric phase arising from on-site elements can be eliminated through a gauge transformation. On the other hand, closing the loop produces a net phase (flux) after tunneling around a closed loop (plaquette). This flux is analogous to a discrete version of Berry's phase and cannot be eliminated through a gauge transformation and it will appear on the tunnel coupling terms in $\mathcal{H}_{\text {eff }}$. In this way, a variety of lattice models can be realized that have nontrivial Peierl's phases.

\section{Band projection and geometric phase}

The arguments above suggest there is a nontrivial Berry flux through a plaquette when energy scales are restricted to a small window around $\omega_{0}$ (and on-site perturbations are sufficiently large). At energies away from $\omega_{0}$ the full degrees of freedom must be considered. The Hamiltonian that describes this general system is given by $\mathcal{H}_{0}=\mathcal{H}_{T}+\mathcal{H}_{\text {os }}$, where $\mathcal{H}_{T}=\sum_{\langle i j\rangle}\left(\boldsymbol{a}_{i}^{\left(\alpha_{i}\right)}\right)^{\dagger} T_{i j} \boldsymbol{a}_{j}^{\left(\alpha_{j}\right)}$ tunnel couples the lattice of cavities defined by $\mathcal{H}_{\mathrm{os}}$ in the previous section. The matrix $T_{i j}$ represents the tunneling between two neighboring sites with on-site mode structure $\alpha_{i}$ and $\alpha_{j}$. The specific form depends on the type of tunneling, but we note that an ideal point contact will have a nonzero overlap for only a single bare cavity mode (not a coupled eigenmode) at frequency $\omega_{0}$. For fundamental cavities this is naturally $a_{0}$, whereas in first excited cavities only one linear combination of $a_{x}$ and $a_{y}$ will have a nonzero contribution to $T_{i j}$. All other tunneling terms will vanish.

The geometric flux can now be rigorously calculated considering a unitary matrix $U_{j}^{(\alpha)}$ at every site $j$ that locally diagonalizes $H_{j}^{(\alpha)}: H_{j}^{(\alpha)}=U_{j}^{(\alpha)} \Delta_{j}^{(\alpha)}\left(U_{j}^{(\alpha)}\right)^{\dagger}$, where $\Delta_{j}^{(\alpha)}$ is a diagonal matrix of on-site energy eigenvalues with a unique mode at frequency $\omega_{0}$. Applying this unitary rotation to every site then transforms the Hamiltonian to $\mathcal{H}_{\mathrm{os}}=$ $\sum_{j}\left(\tilde{\boldsymbol{a}}_{j}^{\left(\alpha_{j}\right)}\right)^{\dagger} \Delta_{j}^{\left(\alpha_{j}\right)} \tilde{\boldsymbol{a}}_{j}^{\left(\alpha_{j}\right)}$ and $\mathcal{H}_{T}=\sum_{\langle i j\rangle}\left(\tilde{\boldsymbol{a}}_{i}^{\left(\alpha_{j}\right)}\right)^{\dagger} \tilde{T}_{i j} \tilde{\boldsymbol{a}}_{j}^{\left(\alpha_{j}\right)}$, where $\tilde{\boldsymbol{a}}_{j}^{\left(\alpha_{j}\right)}=U_{j}^{\left(\alpha_{j}\right)} \boldsymbol{a}_{j}^{\left(\alpha_{j}\right)}$ and $\tilde{T}_{i j}=U_{i}^{\left(\alpha_{i}\right)} T_{i j}\left(U_{j}^{\left(\alpha_{j}\right)}\right)^{\dagger}$. This transformation has the effect of locally diagonalizing each onsite Hamiltonian at the cost of transforming the tunneling matrix between sites.

The restriction to the unique mode at frequency $\omega_{0}$ will amount to a projection into the on-site modes $\hat{a}_{j 0}$. Applying a projection operator $\mathcal{P}_{j 0}$ to eliminate unoccupied modes results in $\mathcal{H}_{\text {eff }, 0} \equiv \sum_{j} \mathcal{P}_{j 0} \mathcal{H}_{0} \mathcal{P}_{j 0}=\sum_{j} \omega_{0} \hat{a}_{j 0}^{\dagger} \hat{a}_{j 0}+$ $\sum_{\langle i j\rangle} \hat{t}_{i j} \hat{a}_{i 0}^{\dagger} \hat{a}_{j 0}$, where the effective tunneling matrix $\hat{t}_{i j}$ can contain a nontrivial phase. We emphasize that while this tunneling phase is gauge dependent, the net flux through a plaquette naturally remains gauge invariant.

We now discuss how the circuit elements described above can result in interesting band structures for a frequency range around $\omega_{0}$. For a plaquette consisting of only fundamental mode cavities, a photon acquires no net phase going around a plaquette (corresponding to zero flux). An $a_{\theta}$ mode cavity would contribute a net flux of $\pi$ to a plaquette if the tunneling contacts cross the nodal line, and 0 otherwise.

For plaquettes with ferrite sites, the TRSB flux is directly proportional to the angle between the two tunneling channels attached to the ferrite cavity. Thus, a resonator with $N$ evenly spaced tunneling contacts will contribute a flux of $\phi=2 \pi / N$ to each adjacent plaquette connected by tunnel couplers. This will allow for simulation of $\alpha=1 / N$ Hofstadter models in different lattice geometries, such as $\alpha=1 / 4$ in a square geometry and $\alpha=1 / 6$ in a triangular geometry. In general, using only the first excited modes we are limited to a total flux of $2 \pi$ per internal ferrite. However, using higher-order modes allows for a net flux of any integer multiple of $2 \pi$ per plaquette.

The nontrivial effective flux is a discrete geometric (Berry's) phase; it is a direct lattice analogue to "synthetic gauge fields" studied in ultracold atomic systems [7]. There, Raman fields are used to couple internal (spin) 

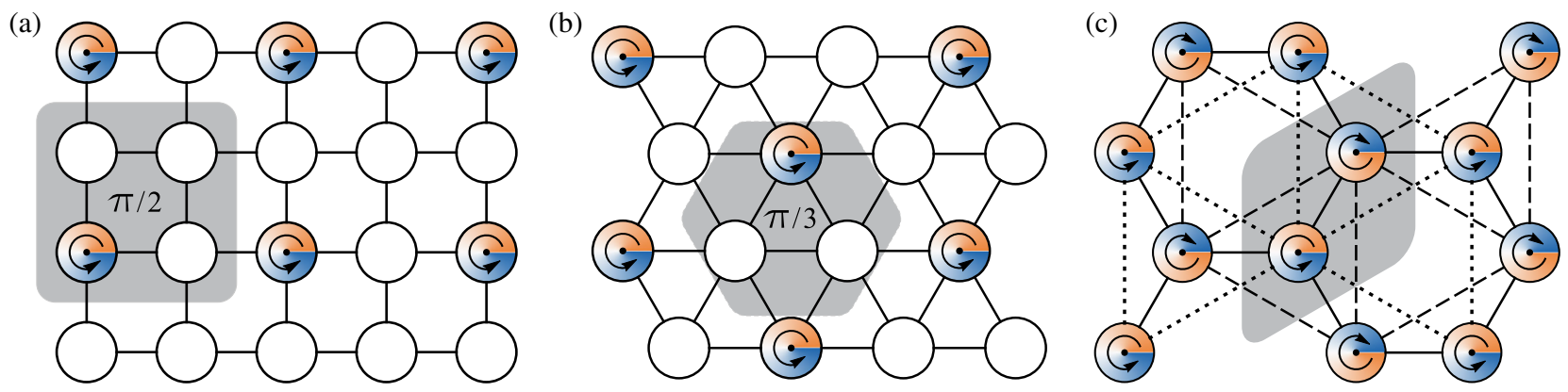

FIG. 3. Implementations of topological band structures described in the main text. (a) Hofstadter model with $\alpha=1 / 4$ : A square, four-site, unit cell has one ferrite and three fundamental resonators. In the effective model near a frequency $\omega_{0}$, the ferrite induces a quarter flux quantum for each of the four neighboring plaquettes. (b) Triangular Hofstadter model with $\alpha=1 / 6$ : A triangular lattice with a three-site unit cell has a single ferrite. This ferrite touches each of six neighboring plaquettes. (c) Haldane model: A hexagonal lattice has a ferrite on each lattice site. By staggering the handedness of the dark modes on A and B sublattices, the net flux vanishes. Addition of next-nearest-neighbor tunneling terms breaks time-reversal symmetry by inducing a flux of $\alpha= \pm \pi / 3$ in each sublattice.

degrees of freedom. The Raman fields vary slowly in space, but also provide a large energetic separation between dressed states. After preparing the system in a single dressed state, the dynamics respond analogously to a system under the influence of a nontrivial external gauge potential. Similar to the study of synthetic gauge fields in ultracold atoms, the microwave lattice scheme can also be extended to the regime of synthetic non-Abelian gauge fields, such as spin-orbit coupling [7,32]; we leave such systems to future work.

\section{Realizing a Hofstadter model}

Given the circuit elements described above, it is now straightforward to construct an $\alpha=1 / 4$ Hofstadter model. Of the many equivalent configurations, we present one that requires the minimum number of ferrites per unit cell.

Consider a square lattice of fundamental resonators, with every fourth fundamental cavity replaced by ferrite cavity, such that all plaquettes include one ferrite. This results in a square four-site unit cell. [See shaded area in Fig. 3(a).] This is consistent with the generic property that $\alpha=p / q$ Hofsdater models have $q$-site unit cells. When the system is probed at frequencies near $\omega_{0}$, the time-reversal symmetrybreaking mode will contribute a phase of $\pi / 2$ into each of the four neighboring plaquettes. Since each plaquette touches only a single ferrite, the total flux will be uniform, $\alpha=(\pi / 2) / 2 \pi=1 / 4$.

We verify that this system provides the expected Hofstadter physics in two ways. First, we explicitly calculate the band projected model for the phase convention defined above. The net flux through a plaquette is found to be $\pi / 2$ as expected. Second, we numerically diagonalize the Hamiltonian including all relevant degrees of freedom. We choose the ferrite-cavity coupling to be 10 times the tunneling energy $\left(g_{F}=10 t\right)$, and consider a strip geometry. As seen in Fig. 4, the full model has six bands. The top and bottom (black) bands are composed almost entirely of the bright magnon modes. These bands are only weakly dispersive, as tunneling between bright modes must occur off resonantly through neighboring fundamental cavities, and is therefore suppressed to $\sim t^{2} / g_{F}$.

In contrast, the middle four (gray) bands are composed almost entirely of dark and fundamental cavity modes. These states form the effective Hofstadter model, and have all expected properties: The four bands are energetically

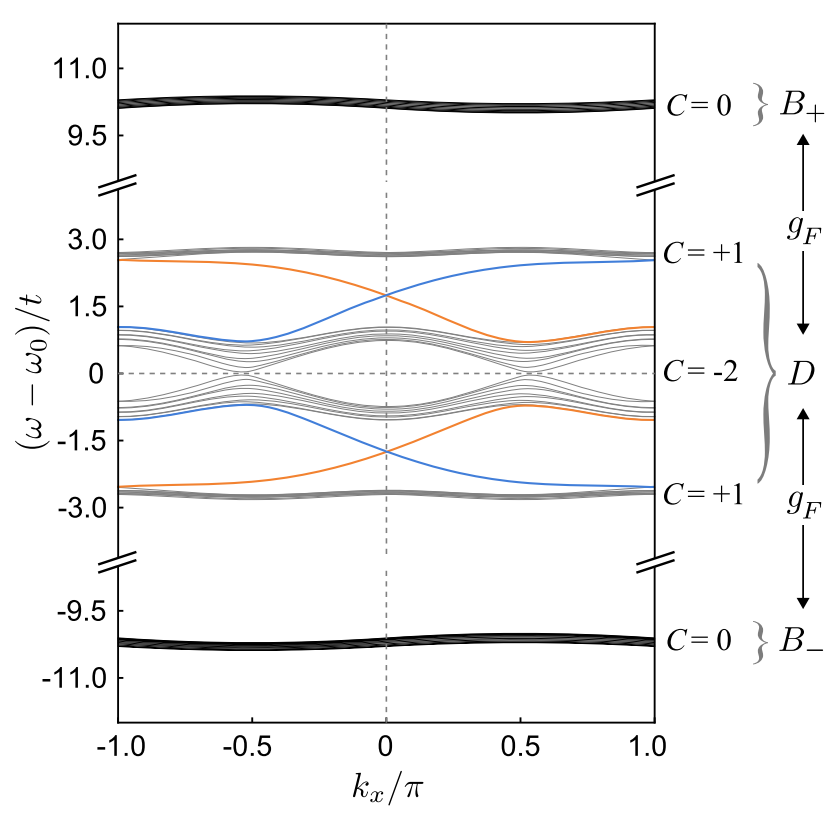

FIG. 4. Band structure of the microwave cavity implementation of the $\alpha=1 / 4$ Hofstdater model in a strip geometry. The unit cell presented in Fig. 3(a) has three fundamental mode cavities, along with a single ferrite site, resulting in a total of six bands. When the ferrite energy is tuned large, two bright bands (black) are far separated from the four bands (gray) near $\omega_{0}$. These four bands have an edge state and Chern number structure consistent with the $\alpha=1 / 4$ Hofstadter model. Here, blue (orange) lines connecting the bulk bands represent edge modes localized on the left (right) edge. No edge states connect the dark bands to the bright bands. 
symmetric around the energy $\omega_{0}$; the top and bottom bands are gapped relative to the two middle bands, which touch at two distinct Dirac points; the band Chern numbers for the continuum model are calculated to be (from top to bottom) $C=0,1,-2,1,0$, consistent with topologically trivial bright bands sandwiching an $\alpha=1 / 4$ Hofstadter model in the dark sector. A finite-size calculation shows chiral edge channels that emerge between the Hofstadter bands, but not between the dark (Hofstadter) and bright bands.

\section{E. Other lattice models}

These circuit elements are powerful for implementing a variety of topological band structures. In passing, we present two additional examples. Figure 3(b) is an implementation of a triangular Hofstadter model with $\alpha=1 / 6$, where we use a triangular lattice with a single ferrite placed in each three-site unit cell. Using arguments similar to above, this introduces a net flux of $\alpha=1 / 6$ per plaquette, as desired.

Tunnel couplings may additionally cross by, e.g., machining channels on both the top and bottom of the substrate supporting the cavity array. This allows for implementation of an even larger class of topologically nontrivial single-particle Hamiltonians. In Fig. 3(c), the Haldane model is constructed from a hexagonal lattice with next-neighbor tunnel couplings added, where a ferrite is added to every site. By alternating the sign of the dc bias field, and thereby the chirality of the ferrite dark modes, between A and B sites, the net flux per plaquette vanishes, while time-reversal symmetry is locally broken. This geometry specifically produces the "ideal" flux configuration that is gauge equivalent to a flux quantum per plaquette [33].

In addition to the examples presented here, other frequently studied Hamiltonians, such as the 2D chiral- $\pi$ [33], or 1D chiral models, such as the SSH model, may also be directly implemented [34]. The circuit architecture described above is useful for realization of a variety of paradigmatic lattice models described in the literature. The possibilities are extensive and we leave elucidation of these to future works.

\section{EXPLORING FRACTIONAL QUANTUM HALL PHYSICS}

The microwave architecture above is linear and should be equally effective at simulating noninteracting topological structures in both the classical and few-photon limits. On the other hand, to create strongly correlated topological states, strong photon-photon interactions are required. It is well established that a nonlinear interaction between photons arises through coupling the microwave cavities to Josephson junction qubits $[28,35]$. The addition of a qubit to each site of the synthetic $\alpha=1 / 4$ Hofstadter model proposed above should be sufficient to explore bosonic fractional quantum Hall physics [9,29] for microwave photons. We expect that the addition of interactions should result in a FQH-like eigenstate lying at the edge of the dark bands, and well separated from the bright bands.

We leave the details regarding the preparation of the many-body eigenstates of our interacting topological Hamiltonian to future work; this is an exciting area of active research, at the interface of many-body physics, dissipation, and nonequilibrium quantum statistical mechanics [36-39]. Nonetheless, for relatively small systems with extremely low loss, it should be possible to spectroscopically populate the (gapped) few-body eigenstate [40]. Such an approach relies on the robustness of the many-body gap to realistic experimental disorder; as will be discussed below, current cavity and qubit technology will allow for a many-body gap that is at least an order of magnitude larger than the largest cavity linewidth, so the FQH manifold should be able to be uniquely prepared and isolated. Once prepared, the cavity can be probed through either single-particle or two-particle correlation measurements [40]. The presence of a Laughlin state can then be verified through a combination of appropriate correlation functions $[18,41]$.

\section{A. Numerical scheme}

We now demonstrate the existence of bosonic FQH eigenstates in our architecture by numerically exploring the combined single-particle and interacting Hamiltonian $\mathcal{H}_{\text {eff,mb }}=\mathcal{H}_{\text {eff }, 0}+\mathcal{H}_{\text {eff }, I}$ projected to describe energies near $\omega_{0}$. Provided the strength of the interactions is weak compared to the ferrite-cavity coupling, a projected qubit-mediated interaction Hamiltonian can be used: $\mathcal{H}_{\text {eff }, I}=\frac{1}{2} \sum_{i} U_{i} \hat{n}_{i 0}\left(\hat{n}_{i 0}-1\right)$, where $U_{i}$ is the effective photon-photon interaction for the photon number $\hat{n}_{i 0}$ of the unique mode near $\omega_{0}$ at site $i$.

We consider a finite-size geometry with $N=N_{x} \times N_{y}$ lattice sites and toroidal boundary conditions, producing a degenerate ground state manifold (GSM). Such boundary conditions need not be a purely theoretical construct: They could be implemented explicitly by either machining the cavities on a physical torus or connecting the opposite boundaries with waveguides [13]. In order to maintain a uniform effective flux, each plaquette must touch exactly one ferrite. This geometric restriction forces $N / 4$ to be an integer, and thus that the projected model has $N_{\phi}=\alpha N=N / 4$ total flux quanta.

In finite-size systems, the incompressibility of the $\mathrm{FQH}$ states will manifest as eigenstates of definite photon number that can be prepared using the spectroscopic techniques discussed above. We therefore consider a system of fixed particle number $N_{p}$, where a FQH ground state is expected to emerge when the filling factor $\nu=N_{p} / N_{\phi}$ is expressible as a certain sequence of rational numbers $[9,10]$. The most stable (largest many-body gap) 
filling is desirable; this occurs at a filling $\nu=1 / 2$, which further restricts $N_{p}=N / 8$ particles ( $N$ divisible by eight). It is experimentally advantageous to start with few-photon states; we thus explore small geometries with $N_{x}=4$ and $N_{p}=2,3,4$ (and, therefore, $N_{y}=2 N_{p}$ ); all numerical results we present below are for $N_{p}=3$ and $N_{y}=6$. (Interestingly, this geometry produces nondispersive bands with $N_{y}=4$, which are only weakly dispersive when $N_{y}=6,8$ [42].)

As explored in previous works $[3,4,42,43]$, many properties of the $\mathrm{FQH}$ are expected to survive in fewparticle geometries. To demonstrate this for our system, we start by exactly diagonalizing $\mathcal{H}_{\text {eff,mb }}$ in the clean limit with $U_{i}=U \gg t$. The geometries described above result in a twofold degeneracy (exact to numerical precision) in the many-body ground state manifold, as expected for a $\nu=1 / 2$ Laughlin state [1,29]. We label the states in the GSM as $\left|\Psi_{1}\right\rangle$ and $\left|\Psi_{2}\right\rangle$; translational invariance allows these states to be distinguished by their center-of-mass momentum [4]. It is useful to impose a twisted boundary condition: $\Psi_{m}\left(x+N_{x}, y\right)=e^{i \gamma_{x}} \Psi_{m}(x, y)$ and $\Psi_{m}\left(x, y+N_{y}\right)=e^{i \gamma_{y}} \Psi_{m}(x, y)$ for all eigenstates $\Psi_{m}(x, y)$, both in the $\operatorname{GSM}(m=1,2)$ and not in the GSM $(m \geq 3)$. The phases $\gamma_{x} \times \gamma_{y}$ represent an AharanovBohm flux $\gamma_{x, y} \in[0,2 \pi)$ adiabatically inserted along the $\hat{x}, \hat{y}$ axes of the torus (as could be implemented with rf-modulated tunneling [20,23]).

Figure 5(a) shows the spectral flow of the lowest 30 eigenstates as a flux quantum is simultaneously inserted along both axes of the torus. Throughout this process the GSM remains gapped. (Here, we include disorder to visually split the two ground states; in the clean limit, the GSM is degenerate to numerical accuracy for all flux values.) For each flux $\gamma$, we define the spectral gap to the first set of excited states:

$$
\Delta_{\mathrm{mb}}(\gamma)=\min _{m \notin \mathrm{GSM}} E_{m}(\gamma)-\max _{m \in \mathrm{GSM}} E_{m}(\gamma),
$$

and we find that the spectral gap is approximately constant for all flux $\boldsymbol{\gamma}=\left(\gamma_{x}, \gamma_{y}\right) \in \mathrm{MBZ}$, where MBZ is the manybody Brillouin zone. Since the GSM remains gapped through the full flux insertion process, we can define the many-body Chern number [44]:

$$
C_{m}=\frac{1}{4 \pi} \int_{\gamma \in \mathrm{MBZ}} d^{2} \gamma\left[\nabla_{\gamma} \times\left\langle\Psi_{m}(\gamma)\left|\nabla_{\gamma}\right| \Psi_{m}(\gamma)\right\rangle\right],
$$

for a state $m \in \mathrm{GSM}$. In a FQH system, the total Chern number in the GSM will evaluate to an integer and give a quantized Hall conductance. For a clean system, both states in the GSM can be identified by their center-of-mass momentum and $C_{m}$ calculated independently. In this case, we find that they each carry a fractionalized many-body Chern number of $C_{1}=C_{2}=1 / 2$, consistent with the thermodynamic limit. This result is also consistent with prior numerical studies of $\mathrm{FQH}$ systems, and an exact solution for a disorder-free finite-size Hofstadter model [42].

In contrast, arbitrarily weak disorder will break translational invariance and remove the degeneracy of the GSM. (This is a finite-size effect, as the degeneracy remains in the thermodynamic limit.) The states in the GSM will remain gapped at all flux values. The calculation of $C_{m}$ is then defined for the $m$ th energy level. We numerically find that, with weak disorder, one state in the GSM randomly obtains $C=1$, while the other has $C=0$, depending on the specific disorder configuration. As the disorder strength is increased, the spectral gap may shrink until the GSM and the first excited set of states are close enough to strongly mix. In this case, the many-body Chern number may mix with the excited state manifold and destroy the FQH state. (Note that level repulsion will prevent the gap from ever exactly vanishing.) In order to quantify the tolerance to disorder, we define the minimum of the many-body gap as

$$
\Delta_{\mathrm{mb}}=\min _{\gamma \in \mathrm{MBZ}} \Delta_{\mathrm{mb}}(\gamma) .
$$

This quantity is used in the next section to study the stability of our scheme.

\section{B. Robustness to disorder}

In any realistic implementation, disorder is present in various forms. We now study the influence of spatial disorder in (1) interaction strength, (2) tunneling energy, (3) flux through each plaquette, and (4) on-site energy upon the interacting Hofstadter model described above. For each disorder type, we calculate the minimum many-body gap, and then average over many disorder configurations. This disorder-averaged many-body gap $\bar{\Delta}_{\mathrm{mb}}$ will be used as a heuristic for the disorder tolerance of the FQH state.

We expect the $\mathrm{FQH}$ state to be robust to disorder in the interaction strength, provided that the average interaction strength is large compared to the tunneling energy. In this blockaded limit, the two-photon wave function overlap is small on any given site, resulting in only a small energy shift due to interaction disorder. (This argument also applies to the continuum case, where the Laughlin wave function has minimal overlap between photons, and, therefore, quenches interaction energy.) This expectation is confirmed through exact diagonalization: The stability of the many-body gap is calculated by taking the smallest gap as a full $2 \pi$ flux is inserted through the twisted boundary conditions, as in Eq. (4). This calculation is performed for a range of average interaction strengths up to $\bar{U}_{i} \sim 20 t$, with a disorder strength $\delta U_{i}=U_{i}-\bar{U}$ uniformly distributed in the different ranges of $\pm \bar{U}\left\{\frac{1}{4}, \frac{1}{2}, \frac{3}{4}, 1\right\}$. The results are shown in Fig. 5(b), demonstrating that interaction disorder does not significantly reduce the many-body gap. We further numerically verify that, for all disorder configurations, the many-body gap remains open throughout flux 
insertion, and the total many-body Chern number in the ground state manifold remains $C=1$.

It is curious that even for the case of the strongest interaction disorder $\left(\max _{i}\left|\delta U_{i}\right|=\bar{U}\right)$, when certain lattice sites can be almost noninteracting, the topological state survives with a many-body gap that does not seem to be limited by the smallest $U_{i}$ in the system. In our system, we still expect only a small probability for the overlap of two photons, and, therefore, a robustness to completely removing some number of qubits. Removing qubits may provide additional flexibility to the proposed experimental realization of the interacting lattice, as the ferrites require a large bias dc magnetic field that is detrimental to the operation of superconducting qubits. We therefore repeat the previous calculation, now with interactions turned off entirely on all ferrite sites [see Fig. 5(c)]. We find that the topological ground state is preserved, although the disorder-averaged many-body gap $\bar{\Delta}_{\mathrm{mb}}$ is reduced by a factor of $\sim 4$.

For interaction disorder, we consider a wide distribution that will realistically arise due to fabrication variations in qubits. By contrast, tunneling disorder is small due to a combination of precision machining techniques and the quadratic insensitivity of the dark state on ferrite sites. We still consider small to moderate disorder in both amplitude $\left(\left|\delta t_{i j}\right|\right.$, uniformly distributed) and phase of the tunneling rates; the disorder in phase translates to a (Gaussian distributed) disordered flux in each plaquette of $\delta \alpha$. The resulting many-body gaps are plotted in Figs. 5(d) and 5(e) when the photon-photon interactions are assumed to be large. We find that the many-body gap decreases only linearly for both tunneling disorder types in this regime, suggesting that tunneling disorder will not be a significant issue in experiment.

The system is more sensitive to on-site disorder, which shifts the on-site mode frequency to $\omega_{0} \rightarrow \omega_{0}+\delta \omega_{i}$. We explore this physics in Fig. 5(f) by adding random on-site (uniformly distributed) disorder in the exact diagonalization calculation. The situation is similar to previous results for disordered (fermionic) FQH systems [45,46]: The total many-body Chern number of the ground state manifold is probabilistically distributed between the lowest-lying states for small disorder. At larger disorder, a collapse of a mobility gap results in a transition to an insulating state. We find that for weak disorder, the many-body gap persists, but declines roughly linearly in the disorder strength. Using 3D microwave cavities, on-site disorder is given by variations in the cavity resonances determined by the cavity dimensions and can be precisely controlled with modern machining techniques. Additionally, the on-site resonance can be made tunable, over a range larger than the tunneling $t$, by, e.g., using a tuning screw or piezostack to perturb critical dimensions of the cavity [47]. Combined with tomography techniques [48,49], which can map out a tight-binding Hamiltonian, on-site disorder can be characterized in the fully coupled lattice, and further reduced.
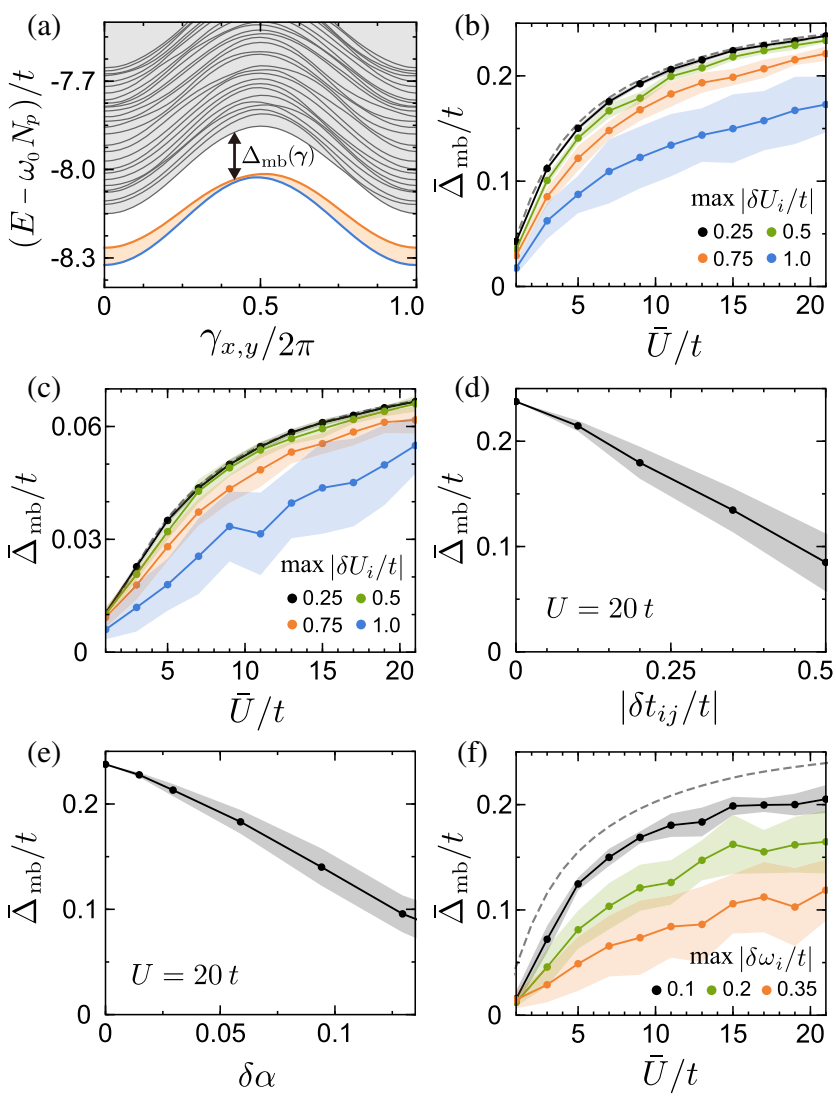

FIG. 5. (a) Spectral flow of a of the lowest 30 many-body eigenstates as a flux $\gamma_{x}=\gamma_{y}=\gamma, \gamma \in[0,2 \pi]$ is inserted into $\mathcal{H}_{\text {eff,mb }}$. Weak disorder is included that visually splits the numerically exact twofold degeneracy of the ground state manifold in the clean limit. For all flux, the GSM (orange and blue lines) remains gapped relative to the (shaded gray) excited states. Provided this gap remains in the presence of disorder, a manybody Chern number can be defined. The disorder-averaged minimum value of this gap is denoted $\bar{\Delta}_{\mathrm{mb}}$. This is used as a metric to test sensitivity to various types of disorder. (b) $\bar{\Delta}_{\text {mb }}$ as a function of the average interaction strength $\bar{U}$ for various strengths of interaction disorder $\delta U_{i}$. The many-body gap is not significantly suppressed at large interaction strengths. (c) Same as (b), but all ferrite sites are made noninteracting. This comes at the cost of a drop of $\bar{\Delta}_{\mathrm{mb}}$ by a factor of about 4 . (d) Effect of disorder in the magnitude of the tunneling strength $\left|\delta t_{i j}\right|$, in a strongly interacting case $\left(U_{i}=20\right)$. (e) Effect of disorder in the flux $\delta \alpha$ through each plaquette. (f) Effect of disorder $\delta \omega_{i}$ in the frequency of the on-site eigenmode near $\omega_{0}$. All error bars (shaded regions) are standard errors of the mean for about 30 realizations of the disorder; the disorders $\delta U_{i},\left|\delta t_{i j}\right|$, and $\delta \omega_{i}$ are uniformly distributed, whereas $\delta \alpha$ is the half width at half maximum of a Gaussian distribution.

\section{DISCUSSION}

We present a scalable 3D microwave circuit architecture to explore bosonic FQH models of photons. Central to this approach is a method for implementing the necessary TRSB flux of the single-particle band structure: the 
on-site mode structure is used to induce this flux, rather than the tunnel couplers themselves. Specifically, a ferrite is used to couple a degenerate cavity manifold, resulting in an isolated cavity eigenmode at frequency $\omega_{0}$ that has uniform phase winding around the cavity periphery. As a photon tunnels in and out, it will accumulate the phase difference between the input and output tunnel contact. This phase is a geometric (Berry's) phase, and will contribute a nontrivial flux to any connected plaquette. This allows for exploration of, e.g., an $\alpha=1 / 4$ Hofstadter model on a square lattice. The circuit elements are entirely passive, providing a distinct advantage from competing protocols that require driving of the tunnel matrix elements.

In practice, $10-\mathrm{GHz}$ microwave resonators may be realized with ferrite time-reversal breaking of order $g_{F} \sim 1 \mathrm{GHz}$ [26]; this allows a Hofstadter model bandwidth of $600 \mathrm{MHz}$ (see Fig. 4,) with tunneling energy of $t=100 \mathrm{MHz}$ and resonator-to-resonator disorder of order $\sim 1 \mathrm{MHz}$. For standard transmon qubits, an interaction strength of $U=350 \mathrm{MHz}$ is readily achieved [50], providing a many-body gap of order (see above) of $\Delta_{\mathrm{mb}} \sim 16 \mathrm{MHz}$. Resonator linewidths of $\kappa \sim 20 \mathrm{~Hz}$ for superconducting coaxial resonators [21] have been achieved in zero field. Realizing such small linewidths in a magnetic field may be challenging, but recent works have achieved $Q \sim 10^{9}$ in microwave cavities with rf field strengths just below $H_{c 1}$ [51]. Since significant timereversal breaking can be achieved at fields below $H_{c 1}$, linewidths in the range of $\kappa \sim 10-100 \mathrm{kHz}$ should be achievable for our system. The large many-body gap and narrow system linewidth will therefore allow the FQH states to be spectroscopically populated [40], and then verified by site-resolved spectroscopy [18,41]. For longlifetime preparation, the system can be coupled to a dissipative bath which drives the system towards the desired number of photons in the ground state [36-39].

This lattice architecture then forms the basis for the investigation of topological many-body physics. Effective photon-photon interactions are implemented through the addition of superconducting qubits. Consistent with previous works, we predict the emergence of a $\nu=1 / 2$ bosonic $\mathrm{FQH}$ eigenstate, even at a large flux per plaquette of $\alpha=1 / 4$. We further verify that this phase is relatively robust to experimentally realistic disorder in on-site energy, interactions, tunneling energy, and flux in a plaquette. In conjunction with state-of-the-art proposals to implement chemical potentials for photons [36-39], this work provides a complete road map to photonic fractional quantum Hall physics, and a path to spectroscopic creation and manipulation of anyons $[52,53]$.

\section{ACKNOWLEDGMENTS}

This work was supported by ARO Grant No. W911NF15-1-0397; D. I. S. acknowledges support from the David and Lucile Packard Foundation; B. M. A. and R.M. acknowledge support from the UChicago MRSEC Grant No. NSF-DMR-MRSEC 1420709; C. O. is supported by the NSF GRFP. The authors thank D. Angelakis, W. DeGottardi, M. Hafezi, and M. Levin for helpful discussions.

[1] H. L. Stormer, D. C. Tsui, and A. C. Gossard, Rev. Mod. Phys. 71, S298 (1999).

[2] A. Stern, Ann. Phys. (Amsterdam) 323, 204 (2008).

[3] E. J. Bergholtz and Z. Liu, Int. J. Mod. Phys. B 27, 1330017 (2013).

[4] N. Regnault and B. A. Bernevig, Phys. Rev. X 1, 021014 (2011).

[5] M. Aidelsburger, M. Atala, M. Lohse, J. T. Barreiro, B. Paredes, and I. Bloch, Phys. Rev. Lett. 111, 185301 (2013).

[6] H. Miyake, G. A. Siviloglou, C. J. Kennedy, W. C. Burton, and W. Ketterle, Phys. Rev. Lett. 111, 185302 (2013).

[7] N. Goldman, G. Juzeliūnas, P. Öhberg, and I. B. Spielman, Rep. Prog. Phys. 77, 126401 (2014).

[8] G. Jotzu, M. Messer, R. Desbuquois, M. Lebrat, T. Uehlinger, D. Greif, and T. Esslinger, Nature (London) 515, 237 (2014).

[9] N. Cooper, Adv. Phys. 57, 539 (2008).

[10] S. Viefers, J. Phys. Condens. Matter 20, 123202 (2008).

[11] M. Greiner, O. Mandel, T. Esslinger, T. W. Hansch, and I. Bloch, Nature (London) 415, 39 (2002).

[12] I. Bloch, J. Dalibard, and W. Zwerger, Rev. Mod. Phys. 80, 885 (2008).

[13] J. Ningyuan, C. Owens, A. Sommer, D. Schuster, and J. Simon, Phys. Rev. X 5, 021031 (2015).

[14] Z. Wang, Y. Chong, J. D. Joannopoulos, and M. Soljacic, Nature (London) 461, 772 (2009).

[15] M. C. Rechtsman, J. M. Zeuner, Y. Plotnik, Y. Lumer, D. Podolsky, F. Dreisow, S. Nolte, M. Segev, and A. Szameit, Nature (London) 496, 196 (2013).

[16] M. Hafezi, S. Mittal, J. Fan, A. Migdall, and J. M. Taylor, Nat. Photonics 7, 1001 (2013).

[17] P. J. J. O’Malley et al., Phys. Rev. X 6, 031007 (2016).

[18] A. A. Houck, H. E. Tureci, and J. Koch, Nat. Phys. 8, 292 (2012).

[19] J. Koch, A. A. Houck, K. L. Hur, and S. M. Girvin, Phys. Rev. A 82, 043811 (2010).

[20] K. Fang, Z. Yu, and S. Fan, Nat. Photonics 6, 782 (2012).

[21] M. Reagor, H. Paik, G. Catelani, L. Sun, C. Axline, E. Holland, I. M. Pop, N. A. Masluk, T. Brecht, L. Frunzio, M. H. Devoret, L. Glazman, and R. J. Schoelkopf, Appl. Phys. Lett. 102, 192604 (2013).

[22] J. Cho, D. G. Angelakis, and S. Bose, Phys. Rev. Lett. 101, 246809 (2008).

[23] L. D. Tzuang, K. Fang, P. Nussenzveig, S. Fan, and M. Lipson, Nat. Photonics 8, 701 (2014).

[24] J. C. Maxwell, Phil. Trans. R. Soc. London 155, 459 (1865).

[25] Y. Tabuchi, S. Ishino, A. Noguchi, T. Ishikawa, R. Yamazaki, K. Usami, and Y. Nakamura, C.R. Phys. 17, 729 (2016).

[26] M. Goryachev, W. G. Farr, D. L. Creedon, Y. Fan, M. Kostylev, and M. E. Tobar, Phys. Rev. Applied 2, 054002 (2014). 
[27] K. Le Hur, L. Henriet, A. Petrescu, K. Plekhanov, G. Roux, and M. Schiró, C.R. Phys. 17, 808 (2016).

[28] M. Reagor, W. Pfaff, C. Axline, R. W. Heeres, N. Ofek, K. Sliwa, E. Holland, C. Wang, J. Blumoff, K. Chou, M. J. Hatridge, L. Frunzio, M. H. Devoret, L. Jiang, and R. J. Schoelkopf, Phys. Rev. B 94, 014506 (2016).

[29] M. Hafezi, A. S. Sørensen, E. Demler, and M. D. Lukin, Phys. Rev. A 76, 023613 (2007).

[30] D. Pozar, Microwave Engineering, 4th ed. (Wiley, New York, 2011).

[31] M. Scully and M. Zubairy, Quantum Optics (Cambridge University Press, Cambridge, England, 1997).

[32] J. Ruseckas, G. Juzeliūnas, P. Öhberg, and M. Fleischhauer, Phys. Rev. Lett. 95, 010404 (2005).

[33] T. Neupert, L. Santos, C. Chamon, and C. Mudry, Phys. Rev. Lett. 106, 236804 (2011).

[34] W. P. Su, J. R. Schrieffer, and A. J. Heeger, Phys. Rev. Lett. 42, 1698 (1979).

[35] A. Wallraff, D. I. Schuster, A. Blais, L. Frunzio, R. S. Huang, J. Majer, S. Kumar, S. M. Girvin, and R. J. Schoelkopf, Nature (London) 431, 162 (2004).

[36] M. Hafezi, M. D. Lukin, and J. M. Taylor, New J. Phys. 15, 063001 (2013).

[37] M. Hafezi, P. Adhikari, and J. M. Taylor, Phys. Rev. B 92 , 174305 (2015).

[38] E. Kapit, M. Hafezi, and S. H. Simon, Phys. Rev. X 4, 031039 (2014).

[39] E. Kapit, J. T. Chalker, and S. H. Simon, Phys. Rev. A 91, 062324 (2015).

[40] P. Schausz, M. Cheneau, M. Endres, T. Fukuhara, S. Hild, A. Omran, T. Pohl, C. Gross, S. Kuhr, and I. Bloch, Nature (London) 491, 87 (2012).
[41] R. O. Umucalilar and I. Carusotto, Phys. Rev. Lett. 108, 206809 (2012).

[42] T. Scaffidi and S. H. Simon, Phys. Rev. B 90, 115132 (2014).

[43] E. Kapit, P. Ginsparg, and E. Mueller, Phys. Rev. Lett. 108, 066802 (2012).

[44] Q. Niu, D. J. Thouless, and Y.-S. Wu, Phys. Rev. B 31, 3372 (1985).

[45] D. N. Sheng, X. Wan, E. H. Rezayi, K. Yang, R. N. Bhatt, and F. D. M. Haldane, Phys. Rev. Lett. 90, 256802 (2003).

[46] X. Wan, D. N. Sheng, E. H. Rezayi, K. Yang, R. N. Bhatt, and F. D. M. Haldane, Phys. Rev. B 72, 075325 (2005).

[47] G. Matthaei, L. Young, and E. Jones, Microwave Filters, Impedance-Matching Networks, and Coupling Structures, Artech House Microwave Library, Vol. 1 (McGraw-Hill, New York, 1964).

[48] D. Burgarth, K. Maruyama, and F. Nori, New J. Phys. 13, 013019 (2011).

[49] R. Ma, C. Owens, A. LaChapelle, D. I. Schuster, and J. Simon, arXiv:1607.05180.

[50] H. Paik, D. I. Schuster, L. S. Bishop, G. Kirchmair, G. Catelani, A. P. Sears, B. R. Johnson, M. J. Reagor, L. Frunzio, L. I. Glazman, S. M. Girvin, M. H. Devoret, and R. J. Schoelkopf, Phys. Rev. Lett. 107, 240501 (2011).

[51] G. Ciovati, P. Dhakal, and G. R. Myneni, Supercond. Sci. Technol. 29, 064002 (2016).

[52] B. Paredes, P. Fedichev, J. I. Cirac, and P. Zoller, Phys. Rev. Lett. 87, 010402 (2001).

[53] R. Umucalilar and I. Carusotto, Phys. Lett. A 377, 2074 (2013). 